\title{
Acute Effects of Mildly Intoxicating Levels of Alcohol on Left Ventricular Function in Conscious Dogs
}

\author{
Che-Ping Cheng, Zak Shihabi, and William C. Little \\ Section of Cardiology, Bowman Gray School of Medicine, Winston-Salem, North Carolina 27103
}

\begin{abstract}
We assessed the effect of alcohol, before and after autonomic blockade, on left ventricular ( $\mathrm{LV}$ ) performance in conscious dogs. 10 animals were instrumented to determine $L V$ volume from ultrasonic $L V$ internal dimensions and measure $L V$ pressure with a micromanometer. The animals were studied in the conscious state after full recovery from the operation. Blood alcohol was undetectable before and $67 \pm 14 \mathrm{mg} / \mathrm{dl}$ (mean \pm SD) at $20 \mathrm{~min}$ after alcohol administration. In response to alcohol, the LV systolic pressure was reduced slightly, the left ventricular end-diastolic pressure increased slightly. The maximum time derivative of $L V$ pressure $\left(d P / d t_{\max }\right)$ and stroke volume were decreased. The end-systolic volume $\left(V_{E S}\right)$, as well as effective arterial elastance, were significantly increased. There was no significant change in heart rate. Variably loaded pressure-volume loops were generated by acute caval occlusion before, immediately, and $20 \mathrm{~min}$ after the intravenous infusion of alcohol $(0.2 \mathrm{~g} / \mathrm{kg})$. Three measures of $\mathrm{LV}$ performance were derived from these variably loaded pressure-volume loops: the end-systolic pressure-volume relation; the stroke work-end-diastolic volume relation; and maximum $\mathrm{d} P / \mathrm{d} t-V_{\mathrm{ED}}$ relation. The slopes of all three relations were significantly decreased in response to alcohol, and all three relations were shifted toward the right, indicating a depression of $\mathrm{LV}$ contractile performance. Similar, but greater depressions of $L V$ performance with alcohol were observed following autonomic blockade. LV performance was restored by infusing dobutamine.

We conclude that mildly intoxicating levels of alcohol (blood concentration $<100 \mathrm{mg} / \mathrm{dl}$ ) are capable of producing $\mathrm{LV}$ contractile depression in conscious animals, which is more marked after autonomic blockade. This suggests that patients with impaired LV function should avoid even small amounts of alcohol. (J. Clin. Invest. 1990. 85:1858-1865.) alcohol • LV function • pressure-volume relation
\end{abstract}

\section{Introduction}

Although the myocardial toxicity of chronic, excessive alcohol consumption is well established (1-5), the acute cardiac effects of occasional social drinking have not been clearly established. Acute, modest alcohol intake has been variably reported to

Address reprint requests to Dr. Cheng, Section of Cardiology, Bowman Gray School of Medicine, 300 South Hawthorne Road, WinstonSalem, NC 27103.

Received for publication 24 August 1989 and in revised form 10 January 1990.

J. Clin. Invest.

(C) The American Society for Clinical Investigation, Inc.

0021-9738/90/06/1858/08 $\$ 2.00$

Volume 85, June 1990, 1858-1865 improve $(6,7)$, to have no direct effect $(8-11)$, or to impair (12-15) left ventricular (LV) performance. Since conventional measures of LV performance are influenced not only by contractile state, but also loading conditions, these conflicting results may be due to the confounding influence of changes in both preload and afterload by alcohol. The influence of loading conditions can be minimized by evaluating LV performance in the pressure-volume plane (16). Such pressure-volume analysis has not been used to assess the effect of alcohol on LV performance.

Some investigators have proposed that a direct myocardial depression due to alcohol may be offset by sympathetic stimulation (17-21). Because of the confounding effects of anesthesia, artificial respiration, and the stress of an acute surgical procedure, this hypothesis cannot be evaluated in anesthetized experimental preparations. The role of the autonomic nervous system in modulating alcohol's effect on LV performance has not been evaluated using load-independent measures of LV performance in conscious animals.

This study was undertaken to assess whether a moderate amount of alcohol produces a depression of $\mathrm{LV}$ contractile performance in normal, conscious animals using load-insensitive measures of $\mathrm{LV}$ performance derived from variably loaded pressure-volume loops. In addition, we used LV pressure-volume analysis to evaluate the role of the autonomic nervous system in modulating alcohol's influence on LV performance.

\section{Methods}

Instrumentation. 10 healthy, adult mongrel dogs (weight $23-30 \mathrm{~kg}$ ) were instrumented as we have previously described (22-25) under halothane anesthesia (1-2\%) induced with rompun ( $1 \mathrm{mg} / \mathrm{lb})$ and sodium thiopental $(6 \mathrm{mg} / \mathrm{kg})$. A sterile, left lateral thoracotomy was performed under general anesthesia with halothane (1-2\%). The pericardium was widely opened. Micromanometer pressure transducers (Konigsberg Instruments, Inc., Pasadena, CA) and polyvinyl catheters for transducer calibration (i.d. $1.1 \mathrm{~mm}$ ) were inserted into the $\mathrm{LV}$ through an apical stab wound and via the left atrial appendage into the left atrium. Three pairs of ultrasonic crystals $(5 \mathrm{MHz})$ were implanted in the endocardium of the LV to measure the anterior-posterior (AP), septal-lateral (SL), and base-apex (long axis, LA) dimensions. All wires and tubing were tunneled subcutaneously and exteriorized through the posterior neck.

Data collection. All studies were performed after full recovery from instrumentation (7-10 d after the original surgery) with the dogs lying on their right sides in a sling. The LV catheter was connected to a pressure transducer (P23Db; Statham Instruments, Oxnard, CA) and calibrated with a mercury manometer. The signal from the micromanometer was adjusted to match that of the catheter. The transit time of $5 \mathrm{MHz}$ sound between the crystal pairs was determined and converted to distance assuming a constant velocity of sound in blood of 1.55 $\mathrm{m} / \mathrm{ms}$.

The analogue signals were recorded on an eight channel oscillograph (MT8500; Astro-Med, West Warwick, RI), digitized with an 
on-line analogue-to-digital converter (Data Translation Devices, Marlboro, MA) at $200 \mathrm{~Hz}$, and stored on a floppy disk memory system utilizing a computer system (PC's Limited, Austin, TX). Each data acquisition lasted for $12 \mathrm{~s}$, spanning several respiratory cycles.

Effect of alcohol. Data were initially recorded with the animals lying quietly on their sides without medication to obtain baseline values. Three sets of variably loaded pressure-volume loops were generated by sudden, transient occlusions of the cavae. This caused a progressive fall in $\mathrm{LV}$ end-systolic pressure, volume, and $\mathrm{d} P / \mathrm{d} t_{\max }$ over a 12 -s recording period (Fig. 1). Immediately after the recording period, the caval occlusion was released and hemodynamic parameters were allowed to restabilize. After all parameters returned to their baseline level, 0.2 $\mathrm{g} / \mathrm{kg}$ body wt alcohol was injected intravenously over $1 \mathrm{~min}$. Both new steady-state recordings and three sets of variably loaded pressure-volume loops were generated immediately, as well as $20 \mathrm{~min}$ after the alcohol infusion.

Interaction of alcohol with autonomic nervous system. To assess the interactions of alcohol with autonomic reflexes, the protocol was repeated after administering hexamethonium $(5 \mathrm{mg} / \mathrm{kg}$ i.v. $)$ and atropine $(0.1 \mathrm{mg} / \mathrm{kg}$ i.v.) on the following day. The adequacy of the autonomic blockade was confirmed in each animal by a lack of an increase in heart rate after arterial pressure was reduced by $>30 \mathrm{mmHg}$ by caval occlusion.

Effect of dobutamine after alcohol. A third protocol was performed in three additional animals. After autonomic blockade was produced with hexamethonium and atropine, data were collected before and again after infusing alcohol (as described above). Then dobutamine $(5 \pm 1 \mu \mathrm{g} / \mathrm{kg}$ per $\mathrm{min}$ ) was infused and data were collected.

Blood ethyl alcohol determination. Blood samples $(5 \mathrm{ml})$ were taken from a different vein than that used for alcohol infusion before, immediately after, and 20 min after alcohol infusion and analyzed by an enzymatic assay (26).

Data analysis. The stored digitized data were analyzed by computer algorithm. Baseline hemodynamic values in each dog were obtained by averaging the data obtained during the 12-s steady state, nonintervention recording periods. End-systole was defined as the upper left-hand corner of the LV pressure-volume loop defined using the iterative technique described by Kono et al. (27). End-diastole was defined as the relative minima following the "a" wave of the high-fidelity LV pressure tracing. End-ejection was defined as peak negative $\mathrm{d} P / \mathrm{d} t$. The $\mathrm{LV}$ volume was calculated as a modified general ellipsoid using the equation: $V_{\mathrm{LV}}=(\mathrm{Pi} / 6) \mathrm{D}_{\mathrm{AP}} \mathrm{D}_{\mathrm{SL}} \mathrm{D}_{\mathrm{LA}}$, where $\mathrm{D}_{\mathrm{AP}}=$ the anterior-posterior $\mathrm{LV}$ dimension, $\mathrm{D}_{\mathrm{SL}}=$ the septal-lateral $\mathrm{LV}$ dimension, and $\mathrm{D}_{\mathrm{LA}}=$ the long axis $\mathrm{LV}$ dimension. We have previously evaluated this method of volume calculation (22-24, 28-30) and it is similar to that used and validated by others (31), except that we determined endocardial dimensions directly, making the subtraction of LV wall thickness or volume unnecessary. This method of volume calculation gives a consistent measure of $\mathrm{LV}$ volume $(r>0.97, \mathrm{SEE}<2 \mathrm{ml})$ despite changes in LV loading conditions, chamber configuration and inotro- pic state. LV stroke work (SW) was calculated by point-by-point integration of the LV pressure-volume loop for each beat as described by Glower et al. (32). The time-derivative of $L V$ pressure $(\mathrm{d} P / \mathrm{d} t)$ was calculated using the 5-point LaGrange method (33). The time constant of relaxation $(T)$ was calculated by fitting $L V$ pressure during isovolumic relaxation to an exponential function with an asymptote $\left(P_{\mathrm{B}}\right)$ : $P=P_{0} \mathrm{e}^{-t / T}+P_{\mathrm{B}}$, where $t=$ time after $\mathrm{d} P / \mathrm{d} t_{\min }, P_{0}, P_{\mathrm{B}}$, and $T$ are constants determined by the data. The arterial elastance was calculated as $P_{\text {ES/SV (34). }}$

Only caval occlusions that produced a fall in LV systolic pressure of at least $30 \mathrm{mmHg}$ were analyzed. Premature beats and the subsequent beat were excluded from analysis. Beats occurring after the heart rate had increased by more than $10 \%$ of initial value were also excluded. The $\mathrm{LV}$ end-systolic pressure $\left(P_{\mathrm{ES}}\right)$-volume $\left(V_{\mathrm{ES}}\right)$ data during the fall of LV pressure produced by each caval occlusion was fit using the least squares technique to: $P_{\mathrm{ES}}=E_{\mathrm{ES}}\left(V_{\mathrm{ES}}-V_{0}\right)$, where $E_{\mathrm{ES}}$ is the slope of the linear $P_{\mathrm{ES}} V_{\mathrm{ES}}$ relation, representing the $\mathrm{LV}$ end-systolic elastance, and $V_{0}$ is the intercept with the volume axis. The volume $\left(V_{100}\right)$ associated with a $P_{\mathrm{ES}}$ of 100 was calculated as: $V_{100}=V_{0}+100 / E_{\mathrm{ES}}$. The $\mathrm{d} P /$ $\mathrm{d} t_{\max }$-end-diastolic volume $\left(V_{\mathrm{ED}}\right)$ and $\mathrm{SW}-V_{\mathrm{ED}}$ relations were quantitated by fitting the data from the same beats from each caval occlusion used to evaluate the $P_{\mathrm{ES}}-V_{\mathrm{ES}}$ relation to: $\mathrm{d} P / \mathrm{d} t_{\max }=\mathrm{d} E / \mathrm{d} t_{\max }\left(V_{\mathrm{ED}}\right.$ $\left.-V_{0, \mathrm{dP} / \mathrm{de}}\right)$ and $\mathrm{SW}=M_{\mathrm{SW}}\left(V_{\mathrm{ED}}-V_{0, \mathrm{SW}}\right)$. The slope of the $\mathrm{d} P / \mathrm{d} t_{\max }-V_{\mathrm{ED}}$ relation, $\mathrm{d} E / \mathrm{d} t_{\max }$, theoretically represents the maximum rate of change of $L V$ elastance $(23,24,29)$. The position of the $\mathrm{d} P / \mathrm{d} t_{\max }-V_{\mathrm{ED}}$ and SW- $V_{\mathrm{ED}}$ relations were calculated by determining the $V_{\mathrm{ED}}$ associated with a dP/dt of $2,000 \mathrm{mmHg} / \mathrm{s}$ and $\mathrm{SW}$ of $2,000 \mathrm{ml} \cdot \mathrm{mmHg}$ :

$V_{2,000, \mathrm{~d} P / \mathrm{d} t}=V_{0, \mathrm{dP} / \mathrm{d} t}+2,000 /\left(\mathrm{d} E / \mathrm{d} t_{\max }\right)$

$V_{2,000, s w}=V_{0, s w}+2,000 / M_{\text {sw }}$.

We used the volumes associated with a $P_{\mathrm{ES}}$ of $100 \mathrm{mmHg}, \mathrm{d} P / \mathrm{d} t_{\max }$ of $2,000 \mathrm{mmHg} / \mathrm{s}$ and $\mathrm{SW}$ of $2,000 \mathrm{ml} \cdot \mathrm{mmHg}\left(V_{100}, V_{2000, \mathrm{dP} / \mathrm{d} t}\right.$, $V_{2,000,5 w}$ ), and not the volume axis intercepts, to quantitate the positions of the relations. The volume axis intercepts are subject to substantial errors since they require extrapolation outside of the range in which the data are available $(16,35,36)$.

Statistical analysis. The slopes, volume intercepts, and positions of the relations $\left(E_{\mathrm{ES}}, V_{0}, V_{100}, \mathrm{~d} E / \mathrm{d} t_{\max }, V_{0, \mathrm{~d} / \mathrm{d} t}, V_{2,000, \mathrm{~d} / \mathrm{d} t}, M_{\mathrm{Sw}}, V_{0, \mathrm{SW}}\right.$, and $V_{2,000,5 w}$ ) for each condition were evaluated as the mean values determined from the three caval occlusions performed under each condition. Results are summarized as the mean $\pm \mathrm{SD}$ and the level of significance was $P<0.05$. Multiple comparisons were performed by analysis of variance. Intergroup comparisons were performed by paired $t$ test with an appropriate correction for the performance of multiple comparison using the Bonferroni inequality (37).

Postmortem evaluation. At the conclusion of the studies, the animals were killed with an overdose of pentobarbital and the hearts were examined to confirm the proper positioning of the instrumentation.

Table I. Effect of Alcohol on Steady-State Hemodynamics without Blockade

\begin{tabular}{|c|c|c|c|c|c|c|c|c|c|c|}
\hline & Heart rate & $P_{\mathrm{ED}}$ & $P_{\text {ES }}$ & $V_{\mathrm{ED}}$ & $V_{\mathrm{EE}}$ & $\mathrm{d} P / \mathrm{d} t_{\max }$ & $\mathrm{d} P / \mathrm{d} t_{\min }$ & sv & $T$ & $E_{\text {a }}$ \\
\hline & $\min ^{-1}$ & $\mathrm{mmHg}$ & $m m H g$ & $m l$ & $m l$ & $\mathrm{mmHg} / \mathrm{s}$ & $m m H g / s$ & $m l$ & $m s$ & $m m H g / m l$ \\
\hline Control & $111 \pm 6$ & $10.2 \pm 1.3$ & $131 \pm 4.1$ & $38.7 \pm 4.3$ & $18.6 \pm 4.6$ & $2886 \pm 248$ & $-2268 \pm 179$ & $20.1 \pm 1.4$ & $37.4 \pm 1.7$ & $8.3 \pm 1.2$ \\
\hline \multicolumn{11}{|l|}{ Alcohol } \\
\hline Immediate & $113 \pm 7$ & $16.7 \pm 3.1^{*}$ & $125 \pm 6.2$ & $34.6 \pm 4.8^{*}$ & $24.1 \pm 4.1^{*}$ & $2577 \pm 237^{*}$ & $-2258 \pm 144$ & $16.5 \pm 1.9^{*}$ & $40.8 \pm 2.1^{*}$ & $10.8 \pm 1.1^{*}$ \\
\hline $20 \mathrm{~min}$ & $110 \pm 3$ & $12.5 \pm 2.4^{*}$ & $120 \pm 4.9$ & $37.9 \pm 5.2$ & $23.9 \pm 3.8^{*}$ & $2657 \pm 251$ & $-2138 \pm 232$ & $14.1 \pm 1.9^{*}$ & $38.9 \pm 4.0$ & $11.0 \pm 0.9^{*}$ \\
\hline
\end{tabular}

$P_{\mathrm{ED}}$, left ventricular end-diastolic pressure $(\mathrm{mmHg}) ; P_{\mathrm{ES}}$, left ventricular end-systolic pressure $(\mathrm{mmHg}) ; V_{\mathrm{ED}}$, left ventricular end-diastolic volume (ml); $V_{\mathrm{EE}}$, left ventricular end-ejection volume $(\mathrm{ml}) ; \mathrm{d} P / \mathrm{d} t_{\max }$, maximum rate of change of left ventricular pressure $(\mathrm{mmHg} / \mathrm{s}) ; \mathrm{d} P / \mathrm{d} t_{\min }$, minimum rate of change of left ventricular pressure $(\mathrm{mmHg} / \mathrm{s}) ; T$, time constant of isovolumic $\mathrm{LV}$ pressure, $E_{\mathrm{a}}$, effective arterial elastance. $* P<0.05$ vs. control. 
Table II. Effect of Alcohol on Steady-State Hemodynamics

\begin{tabular}{|c|c|c|c|c|c|c|c|c|c|c|}
\hline & \multicolumn{10}{|c|}{ Autonomically blocked group } \\
\hline & Heart rate & $P_{\mathrm{ED}}$ & $P_{\mathrm{ES}}$ & $V_{\mathrm{ED}}$ & $V_{\mathrm{EE}}$ & $\mathrm{d} P / \mathrm{d} t_{\max }$ & $\mathrm{d} P / \mathrm{d} t_{\min }$ & SV & $T$ & $E_{a}$ \\
\hline & $\min ^{-1}$ & $\mathrm{mmHg}$ & $\mathrm{mmHg}$ & $m l$ & $m l$ & $\mathrm{mmHg} / \mathrm{s}$ & $\mathrm{mmHg} / \mathrm{s}$ & $m l$ & $m s$ & $m m H g / m l$ \\
\hline Control & $120 \pm 7$ & $12.3 \pm 1.6$ & $127 \pm 8.7$ & $36.8 \pm 4.1$ & $17.6 \pm 2.6$ & $2,327 \pm 197$ & $-2,033 \pm 280$ & $19.2 \pm 1.2$ & $36.8 \pm 1.9$ & $9.1 \pm 1.0$ \\
\hline \multicolumn{11}{|l|}{ Alcohol } \\
\hline Immediate & $121 \pm 8$ & $14.8 \pm 2.7^{*}$ & $122 \pm 6.3$ & $37.5 \pm 3.9$ & $26.6 \pm 3.6^{*}$ & $1,973 \pm 201^{*}$ & $-1,930 \pm 286^{*}$ & $10.9 \pm 1.6$ & $40.9 \pm 2.0^{*}$ & $11.0 \pm 0.9$ \\
\hline $20 \mathrm{~min}$ & $121 \pm 8$ & $14.3 \pm 3.4^{*}$ & $118 \pm 4.6$ & $36.9 \pm 2.9$ & $24.9 \pm 2.8^{*}$ & $2,046 \pm 153^{*}$ & $-1,792 \pm 215^{*}$ & $12.0 \pm 1.1^{*}$ & $40.0 \pm 1.4^{*}$ & $10.6 \pm 1.2$ \\
\hline
\end{tabular}

$* P<0.05$. Abbreviations as in Table I.

\section{Results}

The blood alcohol concentration was undetectable before administering alcohol, and $125 \pm 24.6 \mathrm{mg} / \mathrm{dl}$ immediately after alcohol. $20 \mathrm{~min}$ after alcohol infusion, the blood level was $67 \pm 14 \mathrm{mg} / \mathrm{dl}$.

The steady-state hemodynamic data before and after alcohol infusion are summarized in Tables I and II. In the animals with intact autonomic nervous systems, there was no significant change in heart rate or $P_{\mathrm{ES}}$ in response to alcohol. Both the LV end-diastolic pressure and time constant of isovolumic LV pressure decay were increased immediately after alcohol, while $V_{\text {ED }}$ was reduced. At $20 \mathrm{~min}$ after alcohol, LV end-diastolic pressure was elevated without any significant change in $V_{\text {ED }}$ or the time constant. Similar results were obtained after autonomic blockade, except that the time constant of relaxation was consistently increased after alcohol.

With intact reflexes, $\mathrm{d} P / \mathrm{d} t_{\max }$ was significantly decreased immediately after infusing alcohol, but was not significantly different from control after $20 \mathrm{~min}$. The stroke volume was significantly smaller and $V_{\mathrm{EE}}$ larger after alcohol. The arterial elastance was increased with alcohol. After autonomic blockade, $\mathrm{d} P / \mathrm{d} t_{\max }$ and $\mathrm{SV}$ were uniformly decreased and $V_{\mathrm{EE}}$ increased, but the arterial elastance was unchanged.

A representative analogue recording during a control caval occlusion is shown in Fig. 1. During caval occlusion $P_{\mathrm{ES}}$ fell by $52 \pm 11 \mathrm{mmHg}$ over $17 \pm 3$ beats. These data were used to gener-

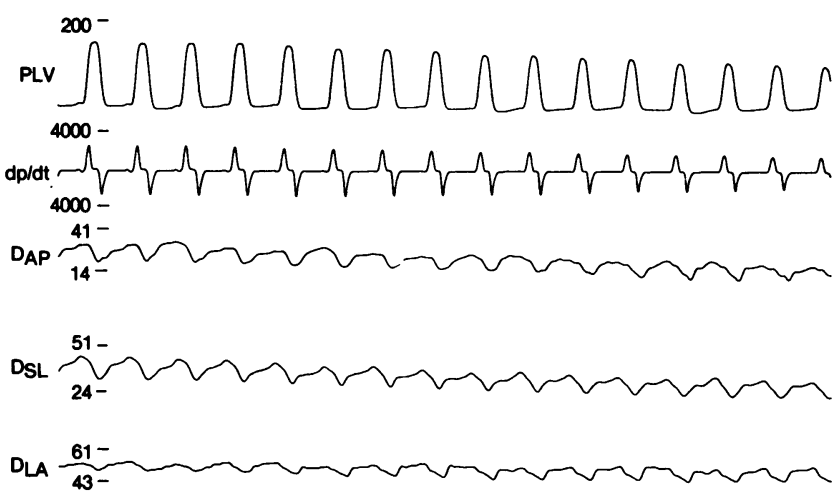

Figure 1. Analogue recording during bicaval occlusion. Bicaval occlusion caused a progressive fall in LV end-systolic pressure, volume, and $\mathrm{d} P / \mathrm{d} t$ over the 12 -s recording period. $P L V=L V$ pressure $(\mathrm{mmHg}), \mathrm{d} P / \mathrm{d} t$ rate of change of PLV $(\mathrm{mmHg}), D_{\mathrm{AP}}=$ anterior-posterior $\mathrm{LV}$ diameter $(\mathrm{mm}) D_{\mathrm{SL}}=$ septal-lacteral diameter $(\mathrm{mm}), D_{\mathrm{LA}}$ $=$ long axis diameter $(\mathrm{mm})$. ate a series of variably loaded pressure-volume loops (Fig. 2). From these loops were derived the $P_{\mathrm{ES}}-V_{\mathrm{ES}}, \mathrm{d} P / \mathrm{d} t_{\max }-V_{\mathrm{ED}}$, and SW- $V_{\text {ED }}$ relations (Figs. 3-5). The slopes of all three relations, $E_{\mathrm{ES}}, \mathrm{d} E / \mathrm{d} t_{\max }, M_{\mathrm{Sw}}$ were significantly $(P<0.05)$ decreased by alcohol (Figs. 3-5, Tables III and IV). Furthermore, all three relations were shifted toward the right, manifested by a significant increase in $V_{100}, V_{2,000, \mathrm{~d} P / \mathrm{d} t}$, and $V_{2,000, \mathrm{sw}}$. These changes were apparent both immediately after alcohol and at $20 \mathrm{~min}$. Similar, but more marked changes in the relations with alcohol occurred after autonomic blockade. Figs. 6 and 7 and Tables III and IV demonstrate these hemodynamic effects of alcohol in the LV pressure-volume plane.

The infusion of the dobutamine reversed the depression of LV performance produced by alcohol (Fig. 8, Table V). All three relations were shifted to the right with a decreased slope in response to alcohol. After dobutamine, all three relations were shifted toward the left with an increase in slope, restoring the relations to their control position and slope.

\section{Discussion}

We investigated the acute effect of alcohol on LV contractile performance in conscious animals. The dose of alcohol used was modest, producing a steady-state blood alcohol concentration $<100 \mathrm{mg} / \mathrm{dl}$, below the usual legal definition of intoxication. We assessed LV contractile performance using variably loaded pressure-volume loops, which helps avoid the potentially confounding effects of changes in loading conditions on conventional measures of $\mathrm{LV}$ performance used in previous studies. All three measures of LV performance that can be derived from the pressure-volume loops (the $P_{\mathrm{ES}}-V_{\mathrm{ES}}, \mathrm{SW}$ $V_{\mathrm{ED}}$, and $\mathrm{d} P / \mathrm{d} t_{\max }-V_{\mathrm{ED}}$ relations) were shifted to the right with a decrease in slope (Figs. 3-5). This indicates that in conscious animals, even modest blood levels of alcohol produce a substantial depression of LV contractile performance.

The depression of LV performance was apparent with intact reflexes, but was more marked after complete autonomic blockade. In addition, alcohol's depression of LV contractile performance could be completely reversed by the infusion of a catecholamine, dobutamine. Thus, it appears that autonomically mediated cardiac stimulation partly offset the direct myocardial depression of alcohol. This effect may be due to central sympathetic stimulation, stimulation of the adrenal medulla or other effects $(17-20,38-40)$.

Our finding of a depression of $\mathrm{LV}$ contractile performance by alcohol in conscious animals is consistent with observations of isolated cardiac muscle $(17,41,42)$. Although some pre- 


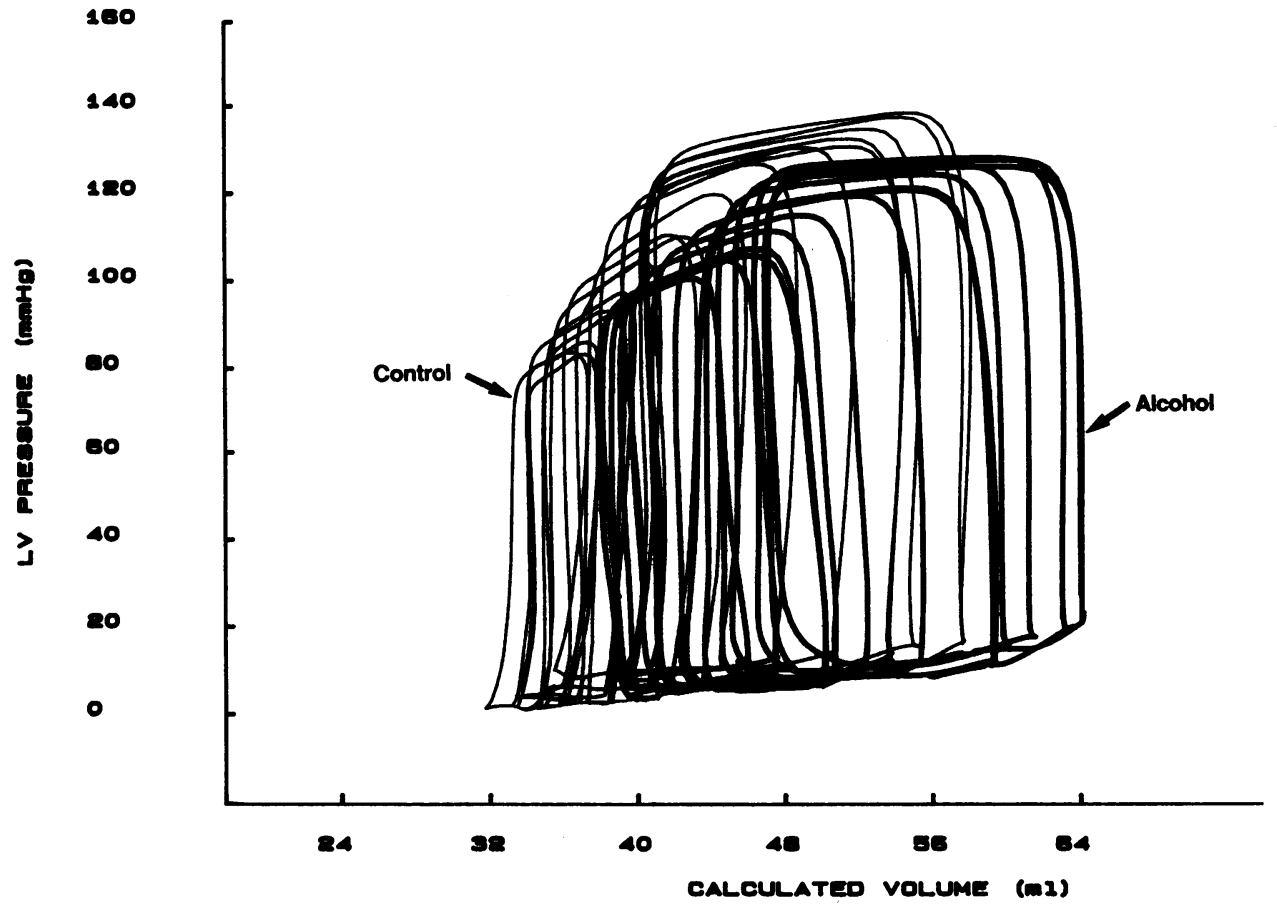

Figure 2. Variably loaded LV pressure-volume loops produced by bicaval occlusion during control and alcohol infusion in a conscious dog. The end-systolic pressure-volume relation is defined by the upperlefthand corner of these loops. vious studies of anesthetized animals $(12,18,43)$, conscious animals (15) and patients (44-46) have suggested that alcohol depresses LV contractile performance, other studies (11-18, 47) have found little or no depression of cardiac performance with alcohol. These conflicting and inconsistent results may

\section{Pes-Ves Relation}

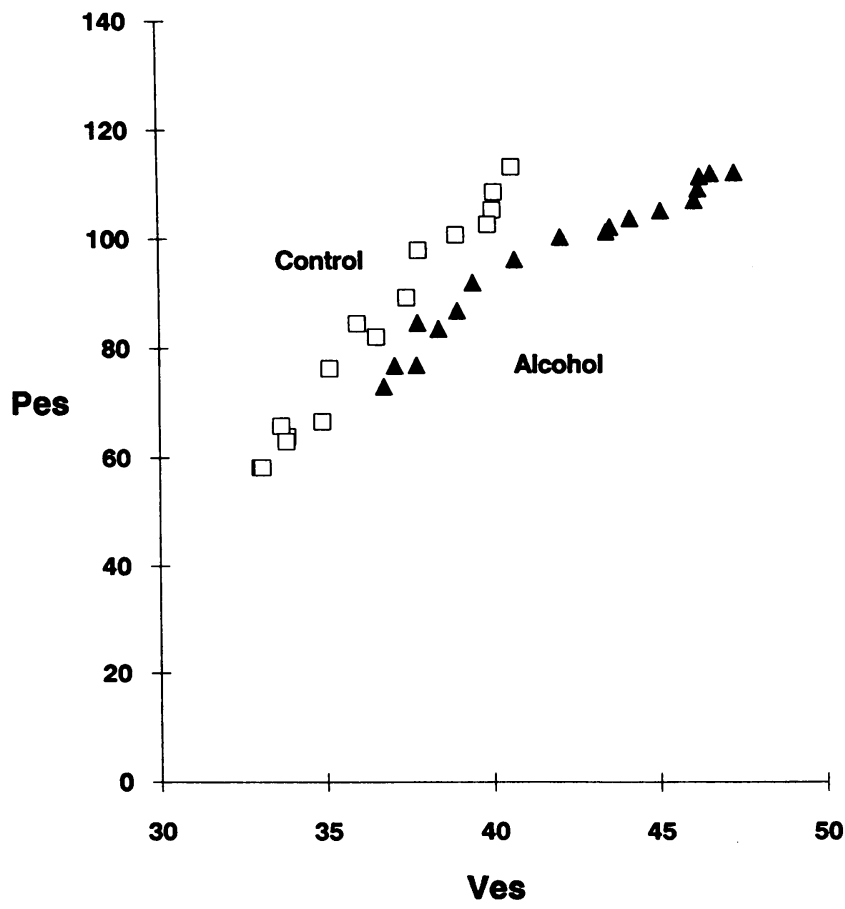

Figure 3. The end-systolic pressure $(P)$-volume $(V)$ relation $\left(P_{\mathrm{ES}}-V_{\mathrm{ES}}\right)$ determined from the loops in Fig. 2. have resulted from the confounding influence of alcohol produced changes in loading conditions on conventional measures of LV performance, and variable effects of autonomic reflexes.

In contrast to previous experimental investigations, we

dP/dt max-Ved Relation

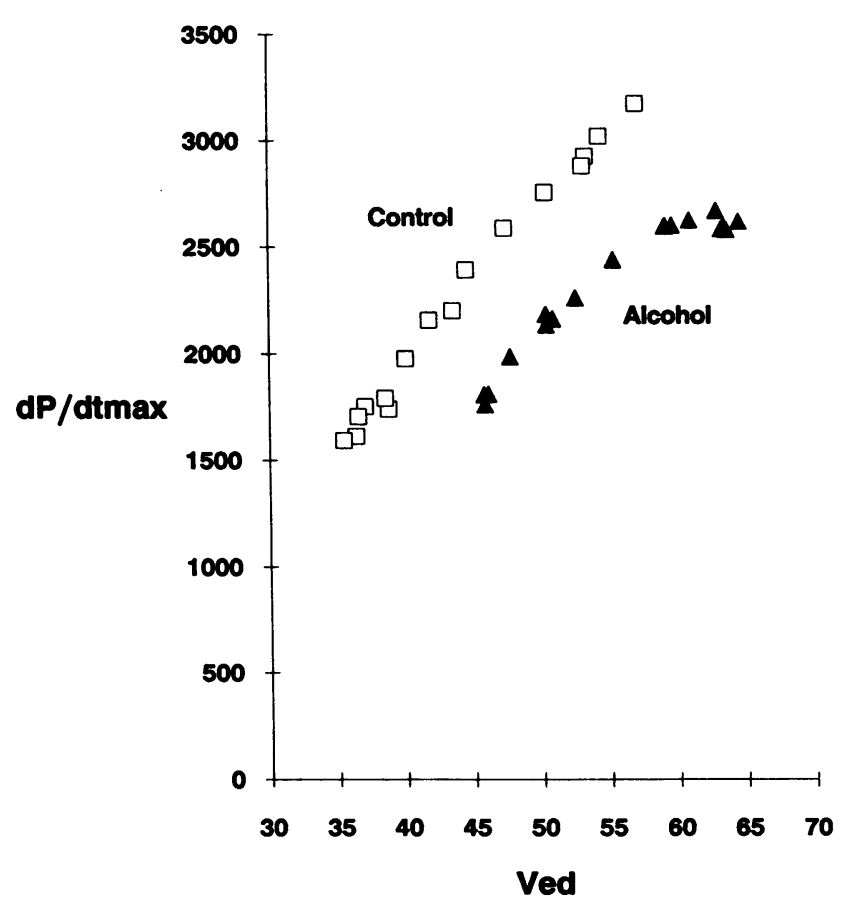

Figure 4. The maximum $\mathrm{d} P / \mathrm{d} t_{\max }$-end-diastolic volume relation ( $\mathrm{d} P /$ $\left.\mathrm{d} t_{\max }-V_{\mathrm{ED}}\right)$ determined from the loops in Fig. 2. 


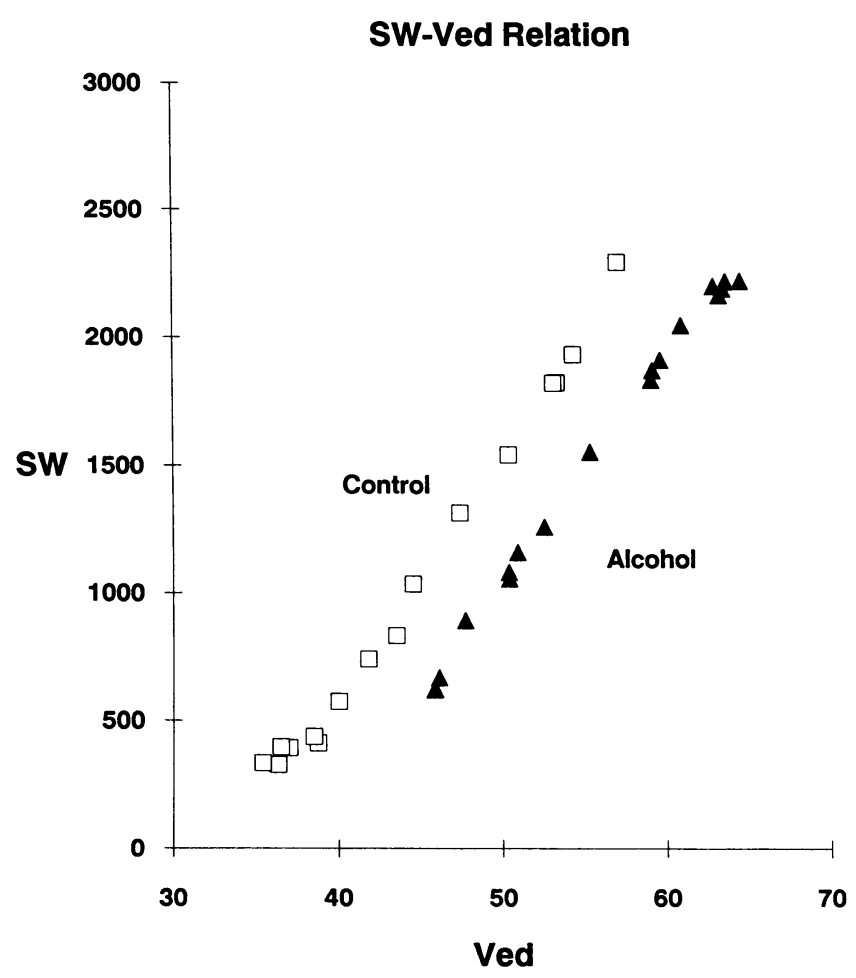

Figure 5. The stroke work end-diastolic volume (SW- $\left.V_{\mathrm{ED}}\right)$ relation, derived from the loops in Figure 2.

evaluated LV performance in the pressure-volume plane. Three measures were used: the $P_{\mathrm{ES}}-V_{\mathrm{ES}}$ relation; the $\mathrm{d} P / \mathrm{d} t_{\max }-$ $V_{\mathrm{ED}}$ relation; and the SW- $V_{\mathrm{ED}}$ relation. Although all three re- lations are derived from variably loaded pressure-volume loops, they potentially provide different information concerning $\mathrm{LV}$ contractile performance $(29,35,48)$. The $P_{\mathrm{ES}}-V_{\mathrm{ES}}$ relation indicates the upper limit of $\mathrm{LV}$ contraction, while the $\mathrm{d} P / \mathrm{d} t_{\max }-V_{\mathrm{ED}}$ relation describes the preloaded corrected maximum rate of pressure development. In contrast, the SW- $V_{\mathrm{ED}}$ relation integrates data from the entire cardiac cycle to describe the external work performed by the LV; thus, it may be influenced by both the systolic and diastolic properties of the $L V$. In addition, the $S W-V_{\mathrm{ED}}$ relation is the most reproducible, and least influenced by altered arterial resistance, but the least sensitive to changes in LV contractile state (47). Each of the three relations responded similarly to alcohol with a rightward shift and a decrease in slope, indicating a depression of contractile performance. Thus, when evaluated by each of these complementary methods, alcohol depressed LV performance.

In addition to alcohol's effect on systolic performance, it also impaired LV diastolic filling. LV end-diastolic pressure was increased by alcohol under all conditions. 20 min after alcohol infusion, during control and after autonomic blockade, this occurred without any increase in $V_{\mathrm{ED}}$, indicating an upward shift of the LV end-diastolic pressure-volume relation. After autonomic blockade, there was also a slowing of relaxation. The alteration of the diastolic LV properties may have contributed to the depression of the LV SW- $V_{\mathrm{ED}}$ relation.

Several potential confounding influences must be taken into account when evaluating the three relations that are derived from $L V P-V$ loops in conscious animals. First, the accuracy of the method of volume measurement must be considered. We have previously determined that the use of three ultrasonically measured LV internal dimensions provides a consistent index of LV volume during caval occlusion despite changes in inotropic state or loading conditions. Second, Su-

Table III. Effect of Alcohol on the $P_{E S}-V_{E S}, d P / d t_{\max }-V_{E D}$, and $S W-V_{E D}$ Relations

\begin{tabular}{|c|c|c|c|c|c|c|c|c|c|}
\hline & \multirow{2}{*}{\multicolumn{3}{|c|}{$P_{\mathrm{ES}}-V_{\mathrm{ES}}$ relation }} & \multicolumn{3}{|c|}{ Without autonomic blockade } & & & \\
\hline & & & & \multicolumn{3}{|c|}{$\mathrm{d} P / \mathrm{d} t_{\max }-V_{\mathrm{ED}}$ relation } & \multicolumn{3}{|c|}{$S W-V_{E D}$ relation } \\
\hline & $E_{\mathrm{ES}}$ & $V_{0, E S}$ & $V_{100, E S}$ & $\mathrm{~d} E / \mathrm{d} t_{\max }$ & $V_{0, \mathrm{dP} / \mathrm{d} t}$ & $V_{2000, d p / d t}$ & $\mathrm{M}_{\mathbf{s w}}$ & $V_{0 . s w}$ & $V_{2,000, \mathrm{sw}}$ \\
\hline Control & $14.7 \pm 2.2^{*}$ & $11.1 \pm 2.3$ & $19.7 \pm 2.8$ & $135.2 \pm 18.5$ & $16.6 \pm 4.6$ & $31.8 \pm 4.9$ & $90.1 \pm 15.9$ & $17.4 \pm 2.9$ & $36.7 \pm 4.5$ \\
\hline \multicolumn{10}{|l|}{ Alcohol } \\
\hline Immediate & $10.6 \pm 1.7^{*}$ & $8.7 \pm 2.3$ & $24.5 \pm 4.1^{*}$ & $97.5 \pm 16.6^{*}$ & $12.1 \pm 4.1$ & $37.5 \pm 4.2^{*}$ & $78.7 \pm 9.2^{*}$ & $17.9 \pm 2.6$ & $39.9 \pm 3.4$ \\
\hline $20 \mathrm{~min}$ & $9.2 \pm 1.3^{*}$ & $7.3 \pm 2.4$ & $24.9 \pm 3.9^{*}$ & $99.1 \pm 15.9^{*}$ & $8.3 \pm 3.4$ & $38.1 \pm 6.3^{*}$ & $85.2 \pm 9^{*}$ & $16.3 \pm 2.1$ & $41.9 \pm 3.7^{*}$ \\
\hline
\end{tabular}

${ }^{*} P<0.01$ vs. control.

Table IV. Effect of Alcohol on the $P_{E S}-V_{E S}, d P / d t_{\max }-V_{E D}$, and $S W-V_{E D}$ Relations

\begin{tabular}{|c|c|c|c|c|c|c|c|c|c|}
\hline & \multirow{2}{*}{\multicolumn{3}{|c|}{$P_{\mathrm{ES}}-V_{\mathrm{ES}}$ relation }} & \multicolumn{3}{|c|}{ Autonomically blocked } & & & \\
\hline & & & & \multicolumn{3}{|c|}{$\mathrm{d} P / \mathrm{d} t_{\max }-V_{\mathrm{ED}}$ relation } & \multicolumn{3}{|c|}{$S W-V_{E D}$ relation } \\
\hline & $E_{\mathrm{ES}}$ & $V_{0, \mathrm{ES}}$ & $E_{100, \mathrm{ES}}$ & $\mathrm{d} E / \mathrm{d} t_{\max }$ & $V_{0, d P / d t}$ & $V_{2,000, d P / d t}$ & $\mathbf{M}_{\text {sw }}$ & $V_{0, s w}$ & $V_{2000,5 w}$ \\
\hline $\begin{array}{l}\text { Control } \\
\text { Alcohol }\end{array}$ & $12.9 \pm 2.8$ & $15.4 \pm 4$ & $21.8 \pm 4.4$ & $134.2 \pm 16.8$ & $11.7 \pm 3.9$ & $28.8 \pm 5.2$ & $86.4 \pm 3.8$ & $26.9 \pm 6.7$ & $28 \pm 6.7$ \\
\hline Immediate & $8.8 \pm 2.4^{* \pm}$ & $12.5 \pm 4.4^{*}$ & $29.4 \pm 5.87^{*}$ & $89.6 \pm 12.4^{* \pm}$ & $9.2 \pm 4.2^{*}$ & $36.8 \pm 7.4^{*}$ & $74.2 \pm 3.8^{*}$ & $24.9 \pm 5.2$ & $29.2 \pm 5.5^{*}$ \\
\hline $20 \mathrm{~min}$ & $8.9 \pm 2.3^{* \pm}$ & $13.3 \pm 4.3^{*}$ & $29.1 \pm 4.9^{*}$ & $115 \pm 20.1^{* \ddagger}$ & $9.8 \pm 3.9^{*}$ & $34.7 \pm 5.8^{*}$ & $72.9 \pm 5.2^{* \neq}$ & $19.6 \pm 4.7$ & $48.5 \pm 5.4^{*}$ \\
\hline
\end{tabular}

${ }^{*} P<0.05$ vs. control. ${ }^{\ddagger} P<0.05$, change specifically different from change without blockade group animals. 


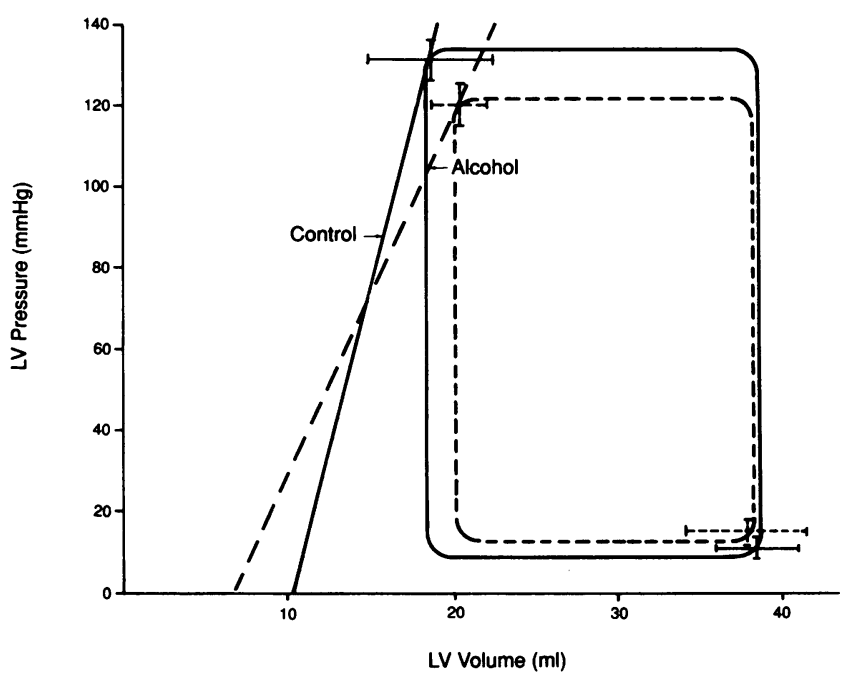

Figure 6. The group (mean \pm SEM) steady-state left ventricular enddiastolic and end-systolic pressures and volumes before and $20 \mathrm{~min}$ after alcohol infusion in the dogs without blockade are shown on the pressure-volume plane along with the end-systolic pressure-volume relations. Note that the end-systolic volume increased and stroke volume decreased despite a decrease in end-systolic pressure.

nagawa et al. have demonstrated that a reduction in coronary perfusion pressure can alter the $P_{\mathrm{ES}}-V_{\mathrm{ES}}$ relation (49). To help avoid this problem, we did not use data points in which $P_{E S}$ was less than $40 \mathrm{mmHg}$. Third, acute reduction of aortic pressure by caval occlusion may activate baroreceptors and cause a reflex increase in inotropic state. This effect becomes apparent after $10 \mathrm{~s}(50)$. We minimized this problem by collecting data for only $12 \mathrm{~s}$ and excluding data points if the heart rate increased by more than $10 \%$ over the initial rate, indicating reflex activation. Further, data were also collected after autonomic blockade. Fourth, the $P_{\mathrm{ES}}-V_{\mathrm{ES}}$ relation can be shifted by factors external to the $\mathrm{LV}$ including changes in the arterial circulation and right ventricular volume $(16,35)$. However, these effects may have less influence on the $\mathrm{d} P / \mathrm{d} t_{\max }-V_{\mathrm{ED}}$ and

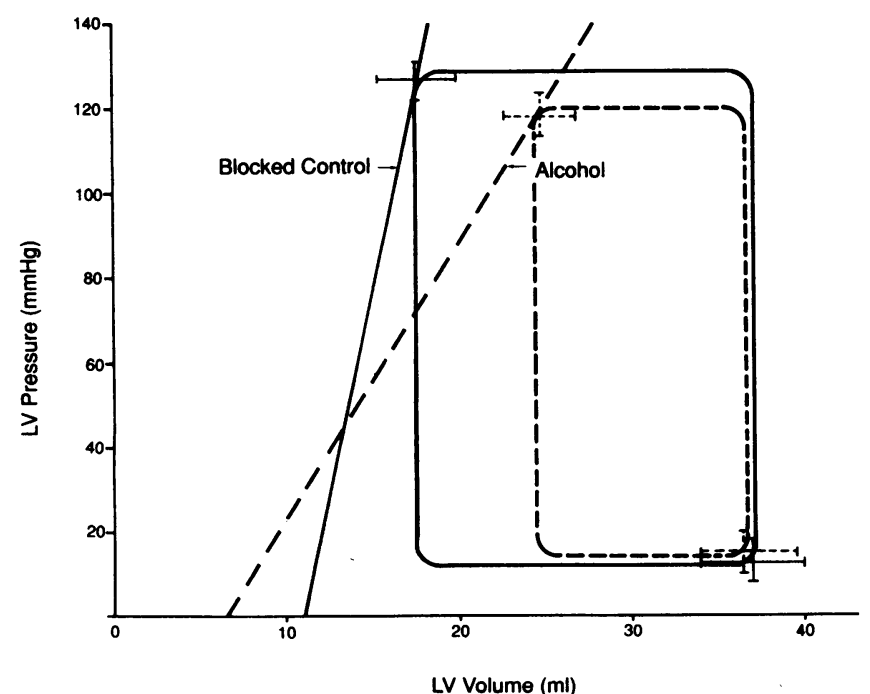

Figure 7. The effect of alcohol following autonomic blockade is shown in the same format as Fig. 4.
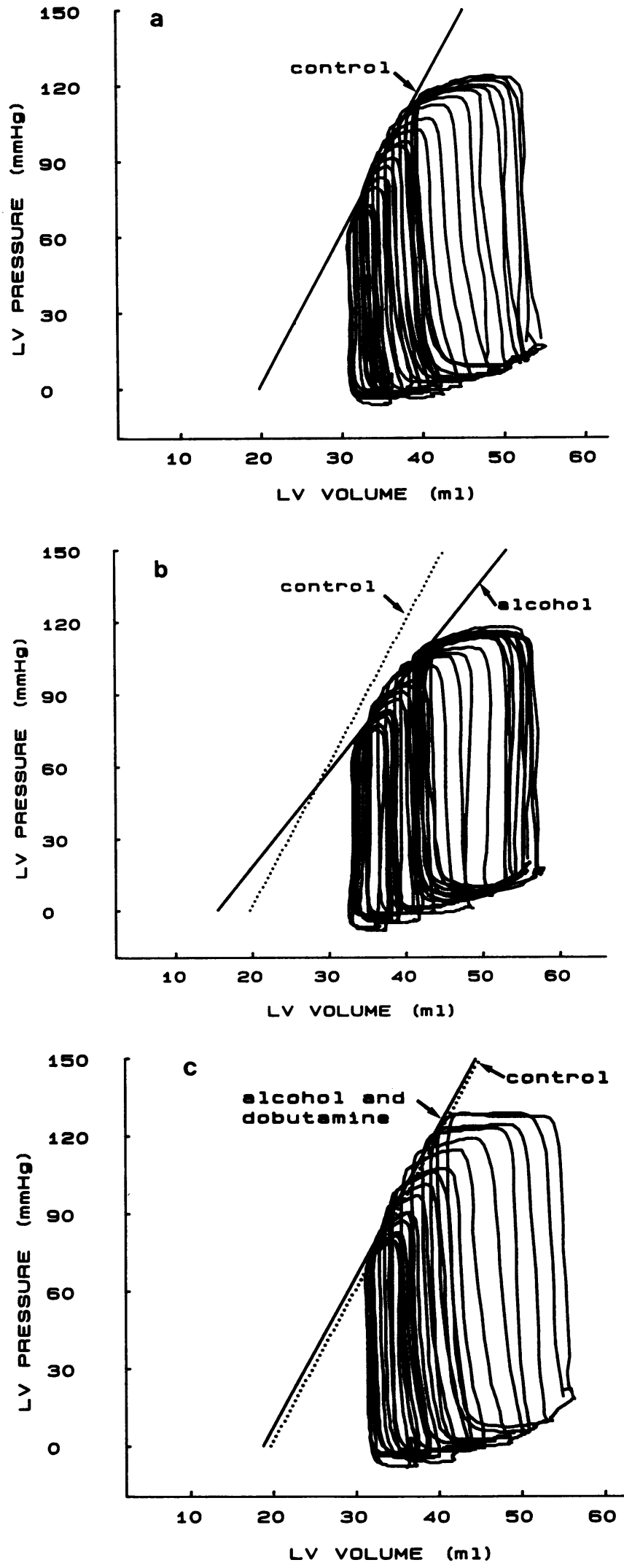

Figure 8 . Variably loaded LV pressure-volume loops and the $P_{\mathrm{ES}}-V_{\mathrm{ES}}$ lines are shown during autonomically blocked control $(a)$, after alcohol $(b)$, and after alcohol and dobutamine $(c)$. The $P_{\mathrm{ES}}-V_{\mathrm{ES}}$ relation is depressed by alcohol (rightward shift, decreased slope) and is restored to control by the infusion of dobutamine. 
Table V. Effect of Dobutamine and Alcohol on the $P_{E S}-V_{E S}, d P / d t_{\max }-V_{E D}$, and $S W-V_{E D}$ Relations

\begin{tabular}{|c|c|c|c|c|c|c|c|c|c|}
\hline & \multicolumn{3}{|c|}{$P_{\mathrm{ES}} V_{\mathrm{ES}}$ relation } & \multicolumn{3}{|c|}{$\mathrm{d} P / \mathrm{d} t_{\max }-V_{\mathrm{ED}}$ relation } & \multicolumn{3}{|c|}{$S W-V_{E D}$ relation } \\
\hline & $E_{\mathrm{Es}}$ & $V_{0, E S}$ & $V_{100, E s}$ & $\mathrm{~d} E / \mathrm{d} t_{\max }$ & $V_{0, \mathrm{dP} / \mathrm{d}}$ & $V_{2,000, \Delta P / d e}$ & $\mathbf{M}_{\mathbf{s w}}$ & $V_{0.5 w}$ & $V_{2000, s w}$ \\
\hline Control & $8.4 \pm 1.7$ & $20.9 \pm 2.8$ & $33.3 \pm 5.0$ & $76.9 \pm 20.7$ & $13.1 \pm 2.8$ & $50.6 \pm 9.1$ & $80.8 \pm 15.1$ & $30.4 \pm 5.4$ & $51.8 \pm 8.0$ \\
\hline Alcohol & $5.3 \pm 1.0^{*}$ & $17.0 \pm 4.1^{*}$ & $36.6 \pm 7.1^{*}$ & $43.7 \pm 15.3^{*}$ & $4.57 \pm 2.0$ & $54.7 \pm 20.3^{*}$ & $73.1 \pm 13.0^{*}$ & $30.6 \pm 5.8$ & $58.6 \pm 9.9^{*}$ \\
\hline $\begin{array}{l}\text { Alcohol and } \\
\text { dobutamine }\end{array}$ & $8.2 \pm 2.2$ & $19.8 \pm 2.8$ & $32.7 \pm 5.7$ & $65.1 \pm 29.7$ & $9.6 \pm 6.6$ & $43.6 \pm 13.2$ & $88.3 \pm 24.2$ & $30.0 \pm 4.4$ & $52.9 \pm 9.75$ \\
\hline
\end{tabular}

${ }^{*} P<0.05$ vs. control.

$\mathrm{SW}-V_{\mathrm{ED}}$ relations (35). Finally, the $P_{\mathrm{ES}}-V_{\mathrm{ES}}$ relation may be curvalinear when evaluated over a wide range $(16,35,36)$. In our study, the relation was determined in similar ranges both before and after alcohol administration; thus, possible curvalinearity should not have affected our result. Furthermore, the depression of $\mathrm{LV}$ performance was also apparent as a rightward shift of the $P_{\mathrm{ES}}-V_{\mathrm{ES}}$ relation and similar responses of both the $\mathrm{d} P / \mathrm{d} t_{\max }-V_{\mathrm{ED}}$ and $\mathrm{SW}-V_{\mathrm{ED}}$ relations.

We administered alcohol intravenously in this study. Thus, we obtained a higher immediate blood level than that of oral ingestion of a similar amount of alcohol. However, the blood level at $20 \mathrm{~min}$ is comparable to that achieved by oral ingestion of a similar amount of alcohol. It has been demonstrated that the manner in which the ethanol was infused (continuous or intermitted) had no influence on the ultimate response (43). Thus, the blood level at $20 \mathrm{~min}$ is comparable to that achieved by oral injection.

The depression of LV contractile performance apparent in conscious animals with alcohol may result from an inhibition of electromechanical coupling at the level of actin-myosin interaction. Such an inhibition has been demonstrated in both skeletal muscle $(51)$ and myocardium $(52,53)$. This may result from a depletion of the calcium stores in the sarcoplasmic reticulum $(52,53)$. The slowed relaxation we observed may be due to altered reuptake of calcium by the sarcoplasmic reticu$\operatorname{lum}(52,53)$.

In conclusion, we found that even mildly intoxicating levels of alcohol are capable of producing direct myocardial depression in conscious animals. This myocardial depression appears to be partially offset by the autonomically mediated effects. Our findings suggest that patients with impaired LV function should avoid even small amounts of alcohol.

\section{Acknowledgments}

The authors gratefully acknowledge Mary Ann Hayner's expert secretarial assistance, the technical assistance of Todd Hall and Bob Rhode, and Dr. Gregory Freeman's review of the manuscript and suggestions.

Supported in part by grants-in-aid from the North Carolina Affiliate of The American Heart Association and the National Institutes of Health (R01-HL-37324). Dr. Little is an Established Investigator of the American Heart Association.

\section{References}

1. Rubin, E. 1979. Seminars in medicine: alcoholic myopathy in heart and skeletal muscle. N. Engl. J. Med. 301:28-33.

2. Ashley, M. J., and J. G. Rankin. 1980. Hazardous alcohol consumption and diseases of the circulatory system. J. Studies Alcohol. 41:1040-1070.
3. Alderman, E. L., and D. J. Coltart. 1982. Alcohol and the heart. Br. Med. Bull. 38:77-80.

4. Friedman, H. S., S. A. Geller, and C. S. Liebe. 1982. Effects of alcohol on the heart muscles. C. S. Liebe, editor. In Medical Disorders: Pathogenesis and Treatment. W. B. Saunders Co., Philadelphia. pp. 436-479.

5. Gould, L., M. Shariff, and M. DiLieto. 1969. Cardiac hemodynamics in alcoholic patients with chronic liver disease and presystolic gallop. J. Clin. Invest. 48:860-868.

6. Gould, L., M. Zahir, A. DeMartino, and R. F. Gomprecht. 1971. Cardiac effects of a cocktail. JAMA (J. Am. Med. Assoc.). 218:17991802.

7. Juchem, S. R., and Klob. 1969. Hemodynamic effects of ethyl alcohol in man. Am. Heart J. 78:133-135.

8. Mierzwiak, D. S., K. Widenthal, and J. H. Mitchel. 1967. Effect of ethanol on the canine left ventricle. Clin. Res. 15:215.

9. Kupari, M., J. Heikkila, and R. Ylikahri. 1983. Acute effects of alcohol on left ventricular dynamics during isometric exercise in normal subjects. Clin. Cardiol. 6:103-108.

10. Riff, D. P., A. C. Jain, and J. T. Doyle. 1969. Acute hemodynamic effects of normal human volunteers. Am. Heart J. 78:592-597.

11. Webb, W. R., D. N. Gupta, W. A. Cook, W. L. Sugg, and F. A. Bashour. 1967. Effects of alcohol on myocardial contractility. Dis. Chest. 52:602-605.

12. Delgado, C. E., N. J. Fortiun, and R. S. Ross. 1975. Acute effects of low doses of alcohol on left ventricular function by echocardiography. Circulation. 51:535-540.

13. Ahmed, S. S., G. E. Levinson, and T. J. Regan. 1973. Depression of myocardial contractility with low doses of ethanol in normal man. Circulation. 48:378-385.

14. Altura, B. M., B. T. Altura, and A. Carella. 1983. Ethanol produces coronary vasospasm: evidence of direct action of ethanol on vascular muscle. Br. J. Pharmacol. 78:260-262.

15. Horwitz, L. D., and J. M. Atkins. 1974. Acute effects of ethanol on left ventricular performance. Circulation. 49:124-128.

16. Kass, D. A., and W. L. Maughan. 1988. From " $E_{\max }$ " to pressure-volume relations: a broader view. Circulation. 77:1203-1212.

17. Nakano, J., and A. V. Prancan. 1972. Effects of adrenergic blockade on cardiovascular responses to ethanol and acetaldehyde. Arch. Int. Pharmacodyn. Ther. 196:259-268.

18. Wong, M. 1973. Depression of cardiac performance by ethanol unmasked during autonomic blockade. Am. Heart J. 86:508-515.

19. Child, T. S., R. B. Kovick, J. A. Levisman, and M. L. Pearce. 1979. Cardial effects of acute ethanol ingestion unmasked by autonomic blockade. Circulation. 59:120-125.

20. Hirsoe, J., R. Higashi, H. Ikeda, K. Tamura, and T. Suzuki. 1973. Effect of ethanol on adrenaline and noradrenaline secretion of the adrenal gland in the dog. Tohuku J. Exp. Med. 109:85-88.

21. Perman, E. S. 1958. The effect of ethyl alcohol on the secretion from the adrenal medulla in man. Acta Physiol. Scand. 44:241-247.

22. Little, W. C., F. R. Badke, and R. A. O'Rourke. 1984. Effect of right ventricular pressure on the LV diastolic pressure-volume relationship in dogs without pericardia. Circ. Res. 54:718-730. 
23. Little, W. C., R. C. Park, and G. L. Freeman. 1987. Effect of regional ischemia and ventricular pacing on $\mathrm{LV} \mathrm{dP/dt} \mathrm{max}_{\max }$-end-diastolic volume relation. Am. J. Physiol. 252:H933-H940.

24. Little, W. C., and G. L. Freeman. 1982. Description of LV pressure-volume relations by time-varying elastance and source resistance. Am. J. Physiol. 253:H83-H90.

25. Freeman, G. L., D. Watkins, and W. C. Little. 1988. A simple method for measurement of left ventricular septal-lateral dimension. Am. J. Physiol. 254:H801-803.

26. Jung, G., and G. Ferard. 1978. Enzyme-coupled measurement of ethanol in whole blood and plasma with a centrifugal analyzer. Clin. Chem. 24:873-876.

27. Kono, A., W. L. Maughan, K. Sunagawa, C. Kallman, K. Sagawa, and M. L. Weisfelt. 1984. Left ventricular end-ejection pressure and peak pressure to estimate the end-systolic pressure-volume relationship. Circulation. 70:1057-1065.

28. Sodums, M. T., F. R. Badke, M. R. Starling, W. C. Little, and R. A. O'Rourke. 1984. Evaluation of left ventricular contractile performance utilizing end-systolic pressure-volume relationships in conscious dogs. Circ. Res. 54:731-739.

29. Little, W. C. 1985. The left ventricular $\mathrm{dP} / \mathrm{dt}_{\max }$-end-diastolic volume relation in closed chest dogs. Circ. Res. 56:808-815.

30. Park, R. C., W. C. Little, and R. A. O'Rourke. 1985. Effect of alterations on the left ventricular activation sequence on the left ventricular end-systolic pressure-volume relation in closed-chest dogs. Circ. Res. 57:706-717.

31. Olsen, C. O., G. S. Tyson, G. W. Maier, J. A. Spratt, J. W. Davis, and J. A. Rankin. 1983. Dynamic ventricular interaction in the conscious dog. Circ. Res. 52:85-104.

32. Glower, D. D., J. A. Spratt, N. D. Snow, J. S. Kabas, J. W. Davis, C. O. Olsen, G. S. Tyson, D. C. Sabiston, Jr., and J. S. Rankin. 1985. Linearity of the Frank-Starling relationship in the intact heart: the concept of preload recruitable stroke work. Circulation. 71:9941009.

Hor. 1987. A comparison of digital algorithms used in computing the derivative of left ventricular pressure. IEEE Trans. Biomed. Engr. (BME). 28:524-529.

33. Marble, A. E., C. M. McIntyre, R. Hastings-James, and C. W. Hor. 1987. A comparison of digital algorithms used in computing the derivative of left ventricular pressure. IEEE Trans. Biomed. Engr. (BME). 28:524-529.

34. Sunagawa, K., W. L. Maughan, and K. Sagawa. 1985. Optimal arterial resistance for the maximal stroke work studied in isolated canine left ventricle. Circ. Res. 56:586.

35. Little, W. C., C. P. Cheng, M. Mumma, Y. Igarashi, J. VintenJohansen, and W. E. Johnston. 1989. Comparison of measures of left ventricular contractile performance from pressure-volume loops in conscious dogs. Circulation. 80:1378-1387.

36. Burkhoff, D., S. Sugiura, D. T. Yue, and K. Sagawa. 1987. Contractility-dependent curvilinearity of end-systolic pressure-volume relations. Am. J. Physiol. 252:H1218-H1227.

37. Glantz, S. A. 1981. Primer of Biostatistics. McGraw-Hill Book Co., New York.
38. Bloom, F. E., G. R. Siggins, S. L. Foote, D. Gruol, G. AstonJones, J. Rogers, Q. Pittman, and D. Staunton. 1984. Noradrenergic involvement in the cellular actions of ethanol. In Catecholamines: Neuropharmacology and Central Nervous System-Theoretical Aspects. Alan R. Liss, New York. 159-167.

39. Mason, S. T., M. E. Corcoran, and H. C. Fibiger. 1979. Noradrenergic processes involved in the locomotor effects of ethanol. Eur. J. Pharmacol. 54:383-387.

40. Perman, E. S. 1960. The effect of ethyl alcohol on the secretion from the adrenal medulla of the cat. Acta Physiol. Scand. 48:323-328.

41. Gimeno, A. L., F. Timenom, and J. L. Webb. 1962. Effects of ethanol on cellular membrane potentials and contractility of isolated rat atrium. Am. J. Physiol. 203:194-196.

42. Fisher, V. J., and F. Valer. 1975. In Alcohol and Abnormal Protein Biosynthesis: Biochemical and Clinical. M. A. Rothschild, M. Oratz, and S. S. Schreiber, editors. Pergamon Press, New York. 187.

43. Mierzwiak, D. S., K. Wildenthal, and J. H. Mitchell. 1972. Acute effects of ethanol on the left ventricle in dogs. Arch. Int. Pharmacodyn. Ther. 199:43-52.

44. Conway, N. 1968. Hemodynamic effects of alcohol in patients with coronary heart disease. Br. Heart J. 30:638-644.

45. Jones, R. D., J. I. Kleinerman, and M. H. Luria. 1975. Observations on left ventricular failure induced by ethanol. Cardiovasc. Res. 9:286-294.

46. Regan, T. J., P. O. Ettinger, B. Haider, S. Ahmed, H. A. Oldewurtel, and M. M. Lyons. 1977. The role of ethanol in cardiac disease. Am. Rev. Med. 28:393-409.

47. Regan, T. J. 1971. Ethyl alcohol and the heart. Circulation. 44:957-963.

48. Kass, D. A., W. L. Maughan, Z. M. Guo, A. Kono, K. Sunagawa, and K. Sagawa. 1987. Comparative influence of load versus inotropic states on indexes of ventricular contractility: experimental and theoretical analysis based on pressure-volume relationships. $\mathrm{Cir}$ culation. 76:1422-1436.

49. Sunagawa, K. W. L., G. Maughan, P. M. S. Friesinger, C. T. S. Chang, and K. Sagawa. 1982. Effects of coronary arterial pressure on left ventricular end-systolic pressure-volume relation of isolated canine heart. Circ. Res. 50:727-734.

50. Kass, D. A., T. Yamazaki, D. Burkhoff, W. L. Maughan, and K. Sagawa. 1986. Determination of left ventricular end-systolic pressure-volume relationships by the conductance (volume) catheter technique. Circulation. 3:586-595.

51. Puszkin, S., and E. Rubin. 1975. Adenosine diphosphate effect on contractility of human muscle actomyosin: inhibition by ethanol and acetaldehyde. Science (Wash. DC). 188:1319-1320.

52. Swartz, M. H., D. I. Repke, A. M. Katz, and E. Rubin. 1974. Effect of ethanol on calcium binding and calcium uptake by cardiac microsomes. Biochem. Pharmacol. 23:2369-2376.

53. Danziger, R. S., M. Sakai, R. G. Hansford, and E. G. Lakatta. 1988. Reversible abolition of cardiac contraction by ethanol is associated with depletion of sarcoplasmic reticulum calcium content. $J$. Am. Coll. Cardiol. II(2):203A. 